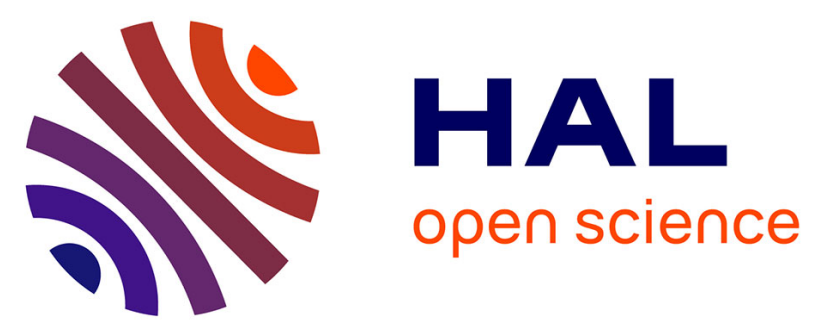

\title{
Coupled thermo mechanical characterisation of polymers based on inverse analyses and IR measurements
}

\author{
Aurelien Maurel-Pantel, Erwan Baquet, Jérôme Bikard, Noëlle Billon
}

\section{To cite this version:}

Aurelien Maurel-Pantel, Erwan Baquet, Jérôme Bikard, Noëlle Billon. Coupled thermo mechanical characterisation of polymers based on inverse analyses and IR measurements. Applied Mechanics and Materials, 2011, 70, pp.393-398. 10.4028/www.scientific.net/AMM.70.393 . hal-00842058

\section{HAL Id: hal-00842058 https://hal.science/hal-00842058}

Submitted on 16 Nov 2014

HAL is a multi-disciplinary open access archive for the deposit and dissemination of scientific research documents, whether they are published or not. The documents may come from teaching and research institutions in France or abroad, or from public or private research centers.
L'archive ouverte pluridisciplinaire HAL, est destinée au dépôt et à la diffusion de documents scientifiques de niveau recherche, publiés ou non, émanant des établissements d'enseignement et de recherche français ou étrangers, des laboratoires publics ou privés. 


\title{
Coupled thermo mechanical characterisation of polymers based on inverse analyses and IR measurements
}

\author{
A.Maurel-Pantel ${ }^{1, \text { a }}$, E. Baquet ${ }^{1}$, J.Bikard ${ }^{1, b}$ and N.Billon ${ }^{1, c}$ \\ ${ }^{1}$ MINES-ParisTech, CEMEF-Centre de Mise en Forme des Matériaux, CNRS UMR 7635, \\ BP 207, 06904 Sophia Antipolis Cedex, France \\ aaurelien.maurel@gmail.com, bjerome.bikard@rhodia.eu.com, ${ }^{c}$ noelle.billon@mines-paristech.fr
}

Keywords: thermo mechanical characterization, semi crystalline polymers, IR measurements, digital image correlation.

\begin{abstract}
Heat dissipation during mechanical testing can disturb experimental characterisation of polymers. In this work it is demonstrated that these effects are not limited to extreme loading conditions such as impacts. A visco-hyperelastic, visco-plastic constitutive model is proposed that accounts for thermo mechanical coupling in a fully 3D thermodynamics approach. Strain-rate and temperature dependencies are coupled using a concept close to the well known time-temperature superposition principle. Constitutive and coupling parameters are identified at the same time using an inverse analysis protocol. An experimental data base is generated for mechanical measurements at different temperatures and strain rates but also for temperatures during tests measured using IR technology. Such a protocol allows investigation on the strain-rate sensitivity in a much more relevant manner than classical one and the value of the so-called Taylor-Quinney coupling parameter is discussed.
\end{abstract}

\section{Introduction}

Nowadays, polymers are more frequently used in industry to create manufactured products for structural applications. As a consequence, effort must be made to mechanically characterise those materials as accurately as possible. The non linear and dissipative visco-elastic behaviour of polymers imposes taking into account strong coupling between mechanical and thermal phenomena as well as complex and combined dependences upon strain-rate and temperature. The classical approach in experimental mechanics of materials does not account for such phenomena in a correct manner and must be revisited.

Nevertheless, these improvements would be useless without relevant constitutive models and protocols for identification of parameters, and it just so happens that most of the existing constitutive models and protocols do not allow model coupling, despite numerous recent approaches.

The present study deals with such improvements, which is combines well instrumented experimental measurements, new generation models and coupled identification for thermal and mechanical parameters. In this paper we illustrate how to combine all these elements to address characterisation of tensile mechanical behaviour of a polyamide 66 .

\section{Thermo mechanical characterisation}

An experimental data base was constructed for a semi-crystalline polyamide 66 according to a protocol based on full-field measurements [1-4]. Samples (Fig. 1a), of rectangular process zone, were machined in $3 \mathrm{~mm}$-thick injection moulded plates. The material was first saturated with humidity in order to ensure that its behaviour was stable during the entire experimental campaign. Tests were performed at temperature ranging from $\mathrm{T}_{\mathrm{g}}-60{ }^{\circ} \mathrm{C}$ to $\mathrm{T}_{\mathrm{g}}+30{ }^{\circ} \mathrm{C}$ and for constant strainrates ranging from $10^{-4} \mathrm{~s}^{-1}$ up to $1 \mathrm{~s}^{-1}$ (using video-controlled tests). Digital image correlation (DIC) on random patterns (painted) was used to analyse the strain field on the front face of the sample. In parallel, temperature at the surface of the process zone was measured using, either an IR pyrometer or an IR camera (FLIR SC5000), on the back and on the side faces of the sample (Fig. 1b). 


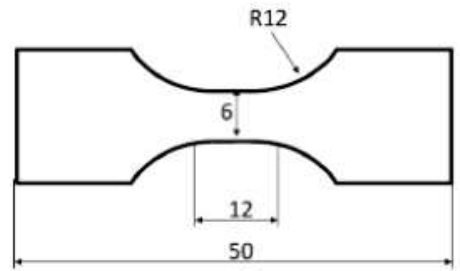

(a)

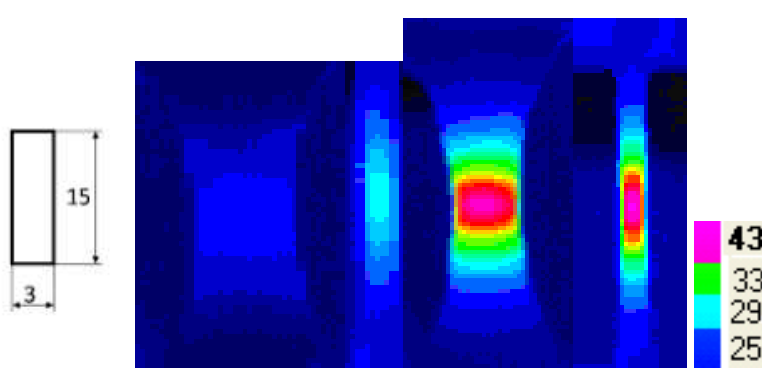

(b)

Figure 1. a) Schematics and dimensions of tensile samples; b) Examples for IR temperature fields on the back (left) and on the side (right) faces of the samples for a $7 \times 10^{-2} \mathrm{~s}^{-1}$ tensile test performed at room temperature. Comparison between two strains of 0.6 and 1.25, respectively (temperatures are given in ${ }^{\circ} \mathrm{C}$ ).

Typical results of IR measurements are depicted in Fig. $1 \mathrm{~b}$ and $2 \mathrm{a}$ for tensions performed at room temperature. Thermo elastic effects were found to induce a $3{ }^{\circ} \mathrm{C}$ decrease in temperature whatever the strain-rate was. Conversely, heating processes were found to be highly dependent upon strainrate. Temperature fields could be superimposed on strain fields and were highly non-uniform on both faces (Fig.1), the hotter zone being restricted to the zone of higher strain. A small difference between the temperatures measured on the back face and those measured on the side face of the sample was observed. This was assumed to be due to the low thermal diffusivity of the polymer. However, differences were so weak compared to longitudinal gradient and compared to the overall increase in temperature during straining that this phenomenon was neglected.

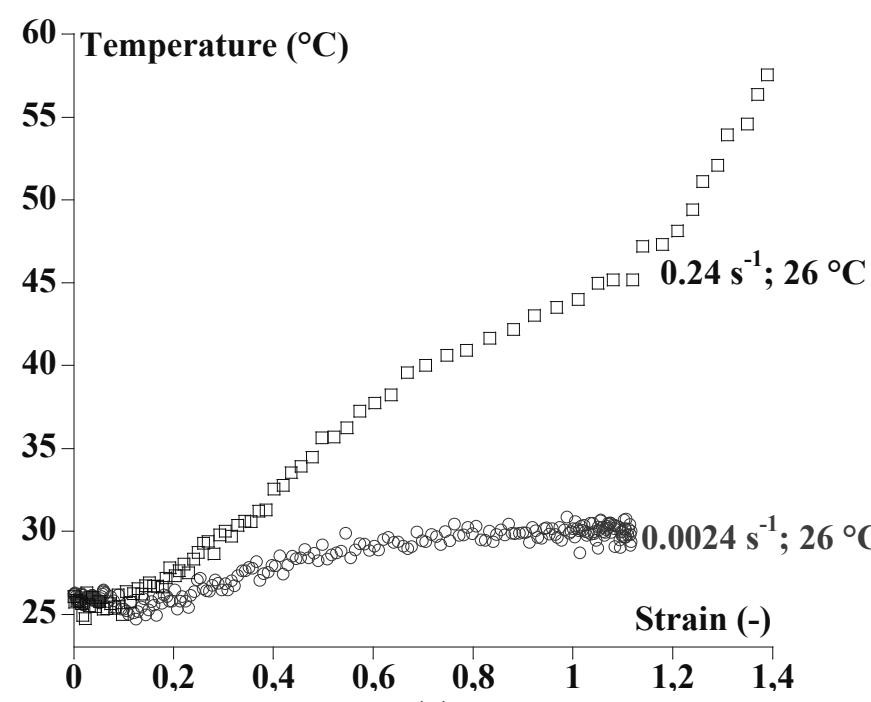

(a)

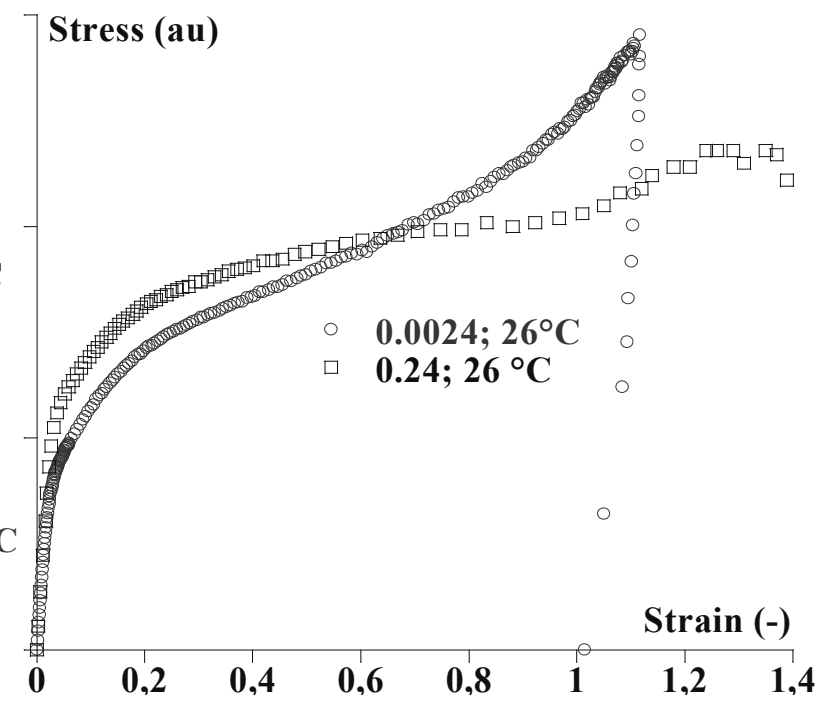

(b)

Figure 2. Comparison between two tensile strain-rates performed at $26{ }^{\circ} \mathrm{C}$; a) Surface temperatures; b) Mechanical curves (mechanical units are arbitrary due to confidentiality).

As a matter of fact, let's emphasise that temperature increased by $60^{\circ} \mathrm{C}$ for strain-rates of $0.5 \mathrm{~s}^{-1}$. This increase in temperature had a real impact on the mechanical behaviour of the polymer and partly counterbalanced the sensitivity to strain-rate of the behaviour and the strain hardening associated to microstructure evolution (Fig. 2). Finally, temperature significantly increased even at low strain-rate (e.g. $+5{ }^{\circ} \mathrm{C}$ for a 0.0024 per second tension) making the accounting for thermomechanical coupling crucially necessary in any case. 


\section{Thermo-mechanical constitutive model [11]}

There are classically two routes to build up constitutive models. One consists in combining elementary processes (elasticity, viscosity, plasticity) via the combination of simple elements (springs, dash pots etc.). See in $[4,6]$ for example. The other route is assuming, within the irreversible processes thermodynamics framework, that stresses derive from energy potentials (See $[2,7,8])$. Despite the fact that both these two routes can be rigorously conducted, accounting for effects of temperature and of strain-rate generally leads to the definition of a lot of arbitrary parameters the values of which are difficult to extract in an unambiguous manner from simple experiments.

To avoid such inconvenience, an alternative approach, developed by Billon et al. [9] in a complete thermodynamic framework, was chosen. Indeed, Billon postulated that microstructure changes are the source of inelasticity in a behaviour that is initially modelled as a hyperelastic network. Those changes are introduced as changes in well chosen internal variables. Inelasticity, per se, is due to the elastic relaxation necessary to balance energy to compensate for variables changes. This model uses Edward-Vilgis' network theory [10] in which stresses derived from an energy potential, w, that can be written either as a function of the three main extensions or as a function of the three invariants (Eq. 1) of the extensions tensor:

$$
\left\{\begin{array}{l}
\mathrm{w}=\frac{\mathrm{kT}}{2}\left[\mathrm{~N}_{\mathrm{s}}\left(\frac{(1+\eta)\left(1-\alpha^{2}\right)}{\left(1-\alpha^{2} \mathrm{I}_{1}^{\mathrm{e}}\right)} \frac{\partial \ln \left(\mathrm{I}_{\eta}^{\mathrm{e}}\right)}{\partial \eta}+\ln \left(\left(1-\alpha^{2} \mathrm{I}_{1}^{\mathrm{e}}\right) \mathrm{I}_{\eta}^{\mathrm{e}}\right)\right)+\mathrm{N}_{\mathrm{c}}\left(\frac{\left(1-\alpha^{2}\right)}{\left.\left.1-\alpha^{2} \mathrm{I}_{1}^{\mathrm{e}} \mathrm{I}_{1}^{\mathrm{e}}+\ln \left(1-\alpha^{2} \mathrm{I}_{1}^{\mathrm{e}}\right)\right)\right]}\right.\right. \\
\mathrm{I}_{\eta}^{\mathrm{e}}=1+\eta \mathrm{I}_{1}^{\mathrm{e}}+\eta^{2} \mathrm{I}_{2}^{\mathrm{e}}+\eta^{3}
\end{array}\right.
$$

where $\mathrm{k}$ is the Boltzman's constant and $\mathrm{T}$ the absolute temperature, respectively. Additionally, $\mathrm{w}$ depends on four variables: $\mathrm{N}_{\mathrm{c}}$ the density of fixed network nodes, $\mathrm{N}_{\mathrm{s}}$ the density of sliding nodes or entanglements, $\alpha$ a parameter ultimately related to chain extensibility and $\eta$ a parameter ultimately representative for level of freedom of entanglements. The stress is given in Eq.(2) and the model is written in the framework of Irreversible Processes Thermodynamics (IPT) and in the frame of large strain approximations:

$$
\sigma=2 F_{e}\left(\frac{\partial w}{\partial I_{1}^{e}} \frac{\partial I_{1}^{e}}{\partial C_{e}}+\frac{\partial w}{\partial I_{2}^{e}} \frac{\partial I_{2}^{e}}{\partial C_{e}}+\frac{\partial w}{\partial I_{3}^{e}} \frac{\partial I_{3}^{e}}{\partial C_{e}}\right) F_{e}^{T}-p^{\prime} I
$$

p' is an arbitrary pressure resulting from the fact that the material is assumed to be incompressible. More precisely, inelasticity results from a change in $\eta$-value the kinetics of which is controlled by the amount of elastic energy stored in the network.

Parameters depend on temperature and strain-rate. To reduce the number of parameters, and according to our experimental observations, it was possible to combine those two dependences in a unique dependence upon equivalent strain-rate at a reference temperature, $T_{\text {ref }}$, as classically defined at low strains for polymers using the usual WLF's approach $[9,11]$. To summarise parameters depends on the fictitious strain-rate:

$$
\dot{\widetilde{\varepsilon}}=a_{T / T_{r e f}} \dot{\varepsilon}_{e q}=a_{T / T_{\text {ref }}} \sqrt{\frac{2}{3} D: D}
$$

where $\mathrm{D}$ is the total strain-rate tensor. $\mathrm{a}_{\mathrm{T} / \mathrm{T}_{\mathrm{ref}}}$ is the so-called shift factor:

$$
\log a_{T / T_{\text {ref }}}=-\frac{C_{1}\left(T-T_{r e f}\right)}{C_{2}+\left(T-T_{r e f}\right)}
$$


$\mathrm{C}_{1}$ and $\mathrm{C}_{2}$ parameters are material characteristics that depend on $\mathrm{T}_{\text {ref }}\left(25^{\circ} \mathrm{C}\right.$ in our case accounting for Tg of the material).

Complementary to that, thermomechanical coupling was introduced in energy equation (Eq. (5)) in a classical manner accounting for dissipation potential, $\phi_{\text {int }}$, related to inelastic strain:

$$
\rho \mathrm{C}_{\mathrm{p}} \dot{\mathrm{T}}=\lambda \Delta \mathrm{T}+\mathrm{T} \frac{\partial \sigma}{\partial \mathrm{T}}: \mathrm{D}_{\mathrm{e}}+\phi_{\text {int }}
$$

This latter is due to disentanglement, which is related to an increase in $\eta$-value according to the model (Eq. (6):

$$
\begin{aligned}
& \phi_{\text {int }}=\mathrm{A}^{\mathrm{D}_{\mathrm{v}}}: \mathrm{D}_{\mathrm{v}}+\mathrm{A}^{\eta} \dot{\eta} \geq 0 \\
& \mathrm{~A}^{\mathrm{D}_{\mathrm{v}}}=2 \mathrm{C}_{\mathrm{e}} \frac{\partial \mathrm{w}}{\partial \mathrm{C}_{\mathrm{e}}}: \mathrm{D}_{\mathrm{v}} \\
& \mathrm{A}^{\eta}=-\frac{\partial \mathrm{w}}{\partial \eta} \dot{\eta}
\end{aligned}
$$

where $D_{v}$ is the inelastic strain-rate tensor. $A^{D v}$ and $A^{\eta}$ are the thermodynamics forces associated to the inelastic deformation process and the entanglements mobility process, respectively. This approach is consistent with Clausius-Duheim inequality and results in a frame that is totally compatible with the Generalized Standard Materials (GSM) approach.

Nevertheless, according to that approach, the inelasticity results from the consumption of some elastic energy to promote changes in the parameter $\eta$. In the case of an athermal process there is no heat dissipation. On the contrary, it can be assumed that a part of the dissipated energy could be transformed into heat. Let's define this energy as a portion of the inelastic energy (as classically done for metals application) using Taylor-Quinney coefficient $\beta$ (7). In this thermo mechanical model, the coefficient is introduced to the intrinsic dissipation in a general manner making no any assumptions concerning its value:

$$
\phi_{\text {int }}=A^{D_{v}}: D_{v}+A^{\eta} \dot{\eta}=\beta\left(D_{e}, a_{T} \dot{\varepsilon}_{e q}\right) A^{D_{v}}: D_{v} \geq 0
$$

where $\beta\left(A^{D v}: D_{v}\right)$ is the energy transformed into heat. Two extreme values for $\beta$ exist:

- $\beta$ close to 1 (but not 1 ): inelasticity is mainly converted into heat;

- $\beta=0$ there is no heat dissipation, inelastic energy is stored in changes in microstructure only. In consequence, the energy equation can be rewritten as Eq. (9):

$$
\rho \mathrm{C}_{\mathrm{p}} \dot{\mathrm{T}}=\lambda \Delta \mathrm{T}+\mathrm{T} \frac{\partial \sigma}{\partial \mathrm{T}}: \mathrm{D}_{\mathrm{e}}+\beta \mathrm{A}^{\mathrm{D}_{\mathrm{v}}}: \mathrm{D}_{\mathrm{v}}
$$

\section{Summary}

The above equations were included in a finite difference code (using a $\theta$-method) to calculate temperature and stress through the central section of the sample. As a first step, strain was assumed to be homogeneous through this section. According to Fig. 1 the temperature was, in parallel, assumed to be uniform trough the width of sample. Consequently, a through thickness 1D-model was enough to simulate the experiments. To take into account the boundary conditions, a convection term with ambient air on the surface was considered. 
To conclud with, this numerical model was associated to an inverse analysis, based on the simplex algorithm available in MATLAB ${ }^{R}$ software. The identification was based on the minimisation of a two objectives cost function that accounted for average axial stress in the section and for surface temperature at this section. This latter was written in a mean-square approach.

In consequence, parameters to be identified were: mechanical parameters $\left(\mathrm{N}_{\mathrm{s}}, \mathrm{N}_{\mathrm{s}}, \alpha\right)$, parameters controlling $\eta$, parameter $\beta$ and their dependence upon $\dot{\widetilde{\varepsilon}}$ (Eq. (3)) and, finally, h, the convection coefficient. The thermal conductivity, the heat capacity and the density of the material were taken from the literature and were assumed to be constant.

Using such an approach, it is possible to reproduce (Fig. 3 and 4) both the mechanical and thermal properties of the polymer over the wide range of experimental sets using only 12 parameters.

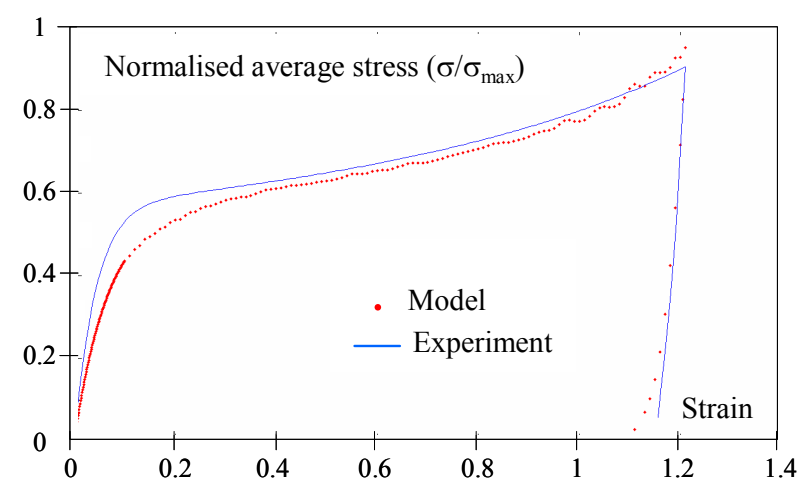

(a)

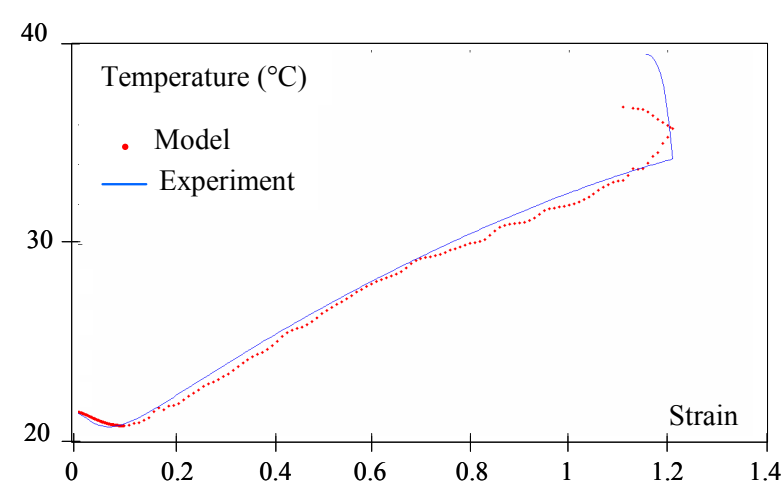

(b)

Figure 3. Comparison between experiments and model for a $0.001 \mathrm{~s}^{-1}$ tensile test; a) Normalised stress vs. strain curve; b) temperature vs. strain.
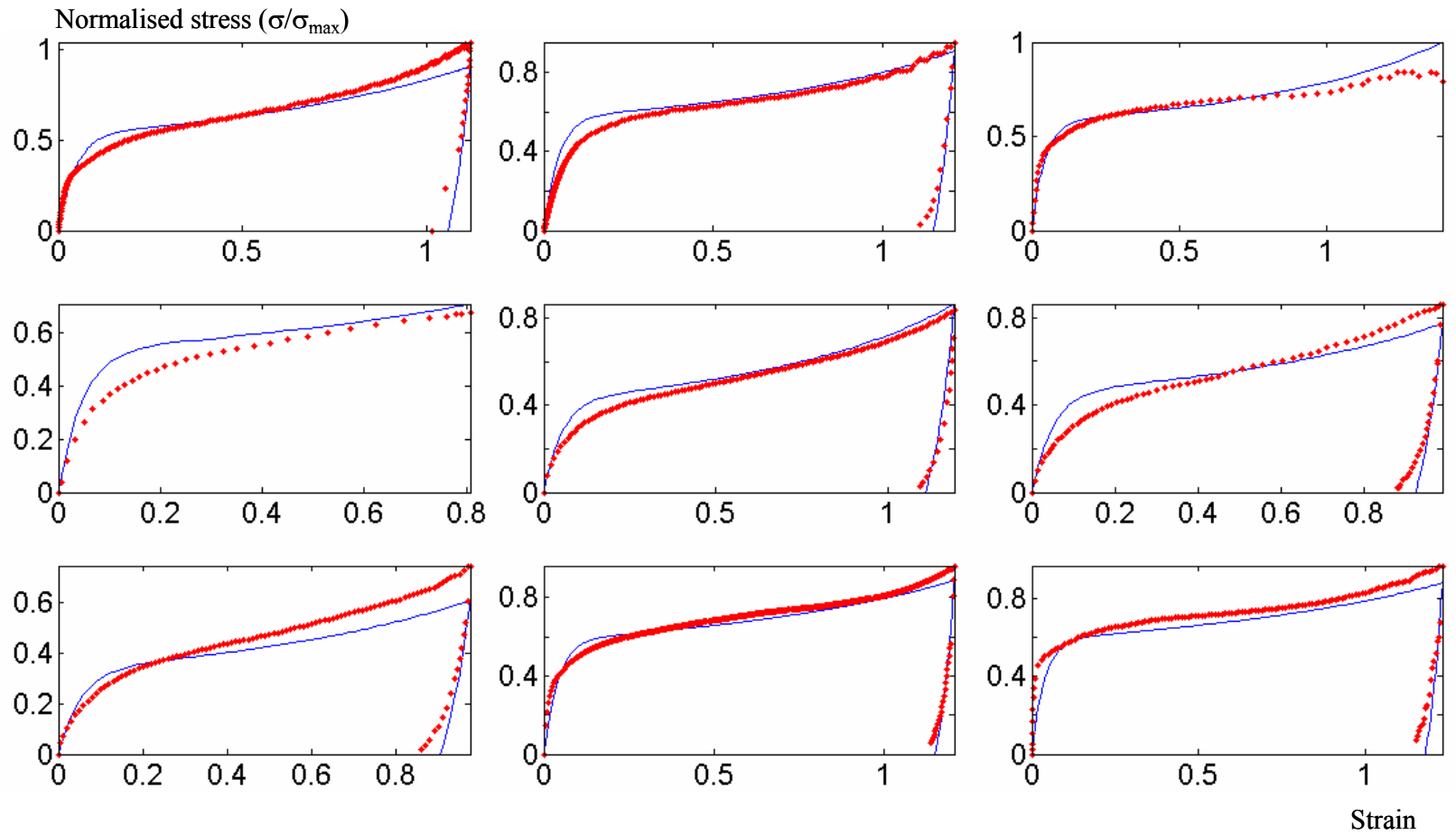

Figure.4. Mechanical curves calculated on the entire range of equivalent strain-rate at the reference temperature (from $10^{-9}$ to $10^{4} \mathrm{~s}^{-1}$ ). Reference stress ( $\sigma_{\max }$ is the same for all conditions). Blue lines are experiments, red dots are calculations. 
Those latter parameters include: dependence upon strain rate and temperature, thermomechanical coupling and convection on the surface of the sample, which is noticeable.

Faster methods are now being developed to improve computation time.

\section{Acknowledgement}

This work has been done in the framework of European project EUREKA 3785, DAMOFIP in partnership with Rhodia, X-Stream and Université de Louvain (UCL).

\section{References}

[1] N. Temimi and N. Billon: Applied Mechanics and Materials Vols. 3-4 (2005), p. 363

[2] N. Temimi-Maaref, A. Burr and N. Billon: Polymer Science, Series A Vol. $50-5$ (2008), p. 558

[3] A. Andriyana, L. Silva and N. Billon: Applied Mechanics and Materials; Advances in Experimental Mechanics Vols. 24-25 (2010), p. 419

[4] A. Andriyana, N. Billon and L. Silva: European Journal of Mechanics A/Solids Vol. 29 (2010), p. 1065

[5] D. Lai, I. Yakimets and M. Guignon: Material Science and Engineering A Vol. 405 (2005), p. 266

[6] E. Roguet, Caractérisation expérimentale et modélisation de l'état relaxé dans le polyamide 11 à hautes temperatures, PhD thesis, ENSMA Poitiers (2006)

[7] A. Drozdov and J. Christiansen: Comput. Mat. Sci. Vol. 39 (2007), p. 729

[8] S. Meo, A. Boukamel, and O. Debordes: Computer and Structure Vol. 80 (2002), p. 2085

[9] N. Billon submitted for publication in J. Appl. Polym. Sci (2010)

[10] S.F. Edwards and T. Vilgis: Polymer Vol.27 (1986), p. 483

[11] E. Baquet, PhD thesis, Mines-ParisTech to be defended (2011)

\section{Glossary}

$\begin{array}{ll}\mathrm{W} & \text { Elastic energy potential } \\ \mathrm{k} & \text { Boltzman's constant } \\ \mathrm{T} & \text { Absolute temperature } \\ \mathrm{D} & \text { Total strain-rate tensor } \\ \mathrm{D}_{\mathrm{e}} & \text { Elastic strain-rate tensor } \\ \mathrm{D}_{\mathrm{v}} & \text { Inelastic strain-rate tensor } \\ \mathrm{F}_{\mathrm{e}} & \text { Elastic extension tensor } \\ \mathrm{C}_{\mathrm{e}} & \text { Cauchy-Green stress tensor } \\ \sigma & \text { Stress tensor } \\ \mathrm{I}_{1}^{\mathrm{e}}, \mathrm{I}_{2}^{\mathrm{e}}, \mathrm{I}_{3}^{\mathrm{e}} & \text { Invariant of elastic extension tensor } \\ \mathrm{A}^{\mathrm{D}_{v}} & \text { Thermodynamic force associated to inelasticity } \\ \mathrm{A}^{\eta} & \text { Thermodynamic force associated to } \eta \\ \beta & \text { Taylor-Quinney coefficient } \\ \mathrm{C}_{\mathrm{p}} & \text { Heat capacity } \\ \lambda & \text { Heat conductivity } \\ \rho & \text { Density }\end{array}$


Advances in Experimental Mechanics VIII

doi:10.4028/www.scientific.net/AMM.70

Coupled Thermo Mechanical Characterisation of Polymers Based on Inverse Analyses and IR Measurements

doi:10.4028/www.scientific.net/AMM.70.393 